\title{
Genetic and environmental correlations between complex phenotypes differ by race/ethnicity and sex
}

Michael Elgart ${ }^{1,2 *}$, Matthew O. Goodman ${ }^{1,2}$, Carmen Isasi ${ }^{3}$, Han Chen ${ }^{4,5}$, Paul S. de Vries ${ }^{4}$, Huichun $\mathrm{Xu}^{6}$, Ani W Manichaikul ${ }^{7}$, Xiuqing Guo ${ }^{8}$, Nora Franceschini ${ }^{9}$, Bruce M. Psaty ${ }^{10}$, Stephen S. Rich ${ }^{11}$, Jerome I. Rotter ${ }^{8}$, Donald M. Lloyd-Jones ${ }^{12}$, Myriam Fornage ${ }^{4,13}$, Adolfo Correa ${ }^{14}$, Nancy L. HeardCosta $^{15,16}$, Ramachandran S. Vasan ${ }^{15,17}$, Ryan Hernandez ${ }^{18}$, Robert C. Kaplan ${ }^{3,19}$, Susan Redline ${ }^{1,2}$, the Trans-Omics for Precision Medicine (TOPMed) Consortium, Tamar Sofer ${ }^{1,2,20^{*}}$

${ }^{1}$ Division of Sleep and Circadian Disorders, Brigham and Women's Hospital, Boston, MA, USA

${ }^{2}$ Department of Medicine, Harvard Medical School, Boston, MA, USA

${ }^{3}$ Department of Epidemiology and Population Health, Albert Einstein College of Medicine, Bronx, NY, USA

${ }^{4}$ Human Genetics Center, Department of Epidemiology, Human Genetics, and Environmental Sciences, School of Public Health, The University of Texas Health Science Center at Houston, Houston, TX, USA

${ }^{5}$ Center for Precision Health, School of Biomedical Informatics, The University of Texas Health Science Center at Houston, Houston, TX, USA

${ }^{6}$ Department of Medicine, University of Maryland School of Medicine, Baltimore, MD, USA

${ }^{7}$ Center for Public Health Genomics, University of Virginia, VA, USA

${ }^{8}$ The Institute for Translational Genomics and Population Sciences, Department of Pediatrics, The Lundquist Institute for Biomedical Innovation at Harbor-UCLA Medical Center, Torrance, CA, USA

${ }^{9}$ Department of Epidemiology, University of North Carolina, Chapel Hill, NC, USA

${ }^{10}$ Cardiovascular Health Research Unit, Departments of Medicine, Epidemiology, and Health Services, University of Washington, Seattle, WA, USA

${ }^{11}$ Center for Public Health Genomics, University of Virginia School of Medicine, Charlottesville, VA, USA

${ }^{12}$ Department of Preventive Medicine, Northwestern University, Chicago, IL, USA

${ }^{13}$ Brown Foundation Institute of Molecular Medicine, McGovern Medical School, University of Texas Health Science Center at Houston, Houston, TX, USA

${ }^{14}$ Department of Population Health Science, University of Mississippi Medical Center, Jackson, MS, USA

${ }^{15}$ Boston University and National Heart Lung and Blood Institute's Framingham Heart Study, Framingham, MA, USA

${ }^{16}$ Department of Neurology, Boston University School of Medicine, Boston, MA, USA

${ }^{17}$ Preventive Medicine \& Epidemiology, and Cardiovascular Medicine, Medicine, Boston University School of Medicine, and Epidemiology, Boston University School of Public health, Boston, MA, USA

${ }^{18}$ Department of Bioengineering and Therapeutic Sciences, University of California, San Francisco, CA, USA

${ }^{19}$ Fred Hutchinson Cancer Research Center, Division of Public Health Sciences, Seattle, WA, USA

${ }^{20}$ Department of Biostatistics, Harvard T.H. Chan School of Public Health, Boston, MA, USA

${ }^{*}$ Correspondence:

Michael Elgart melgart@bwh.harvard.edu;

Tamar Sofer tsofer@bwh.harvard.edu 
medRxiv preprint doi: https://doi.org/10.1101/2021.09.05.21263126; this version posted September 10, 2021. The copyright holder for this preprint (which was not certified by peer review) is the author/funder, who has granted medRxiv a license to display the preprint in perpetuity.

It is made available under a CC-BY-NC-ND 4.0 International license .

\section{Abstract}

We developed novel closed-form estimators of genetic and environmental correlation coefficients. We applied them to estimate over 4,000 genetic and environmental correlations between multiple phenotypes in a diverse sample from the Trans-Omics in Precision Medicine (TOPMed) program. We found substantial differences in heritabilities, genetic, and environmental correlations of multiple phenotypes and phenotype-pairs between Black, Hispanic/Latino and White populations as well as between sexes. Finally, we quantified genetic and environmental correlations between phenotypic domains, each characterized by multiple phenotypes. Altogether we provide a novel, indepth framework for examining relations among complex human phenotypes and their determinants.

\section{List of Abbreviations}

BBJ - BioBank Japan; UKB - UK Biobank; GREML - genetic restricted maximum likelihood analysis; GWAS - genome-wide association study; LD - linkage disequilibrium; LDSC - linkage disequilibrium score regression; HE - Haseman-Elston regression; FDR - False Discovery Rate; TOPMed - TransOmics in Precision Medicine ; HCHS/SOL - Hispanic Community Health Study / Study of Latinos; BMI - body-mass index; HDL - high-density lipoprotein; WBC - white blood cell counts; CRP - CReactive Protein; RBC - red blood cells; AHI - Apnea Hypopnea Index;

\section{Introduction}

Both genetics and environment determine human phenotypes and the correlations between them $[1,2]$. Correlations can arise due to multiple forms of causal relationships including common genetic and environmental determinants, (bi)directional causal associations and others. The correlations between phenotypes can reveal genetic architecture, help uncover gene functions and disease mechanisms, improve diagnosis and aid in therapeutic interventions [3]. Given appropriate data, 
medRxiv preprint doi: https://doi.org/10.1101/2021.09.05.21263126; this version posted September 10, 2021. The copyright holder for this

phenotypic correlations can be decomposed into genetic and environmental components by estimating corresponding measures, such as genetic correlation [4].

Several studies have leveraged the data generated by large studies with genotyped individuals and multiple measured phenotypes (e.g. BioBank Japan (BBJ)[5] and UK Biobank (UKB)[6]), to estimate genetic correlations between various phenotypes [7-10]. Dozens of pairwise correlations between phenotypes were estimated and reported with the vast majority of participants being of the same race/ethnicity of either European or East Asian descent[9, 10] and of mixed genders. Race/ethnicity and gender, are complex constructs, associated with differences in phenotypic distributions between different subpopulations due, to some extent, to both underlying genetics and different environmental exposures [11-13]. The genetic background of a subpopulation manifests in allele frequencies, effect sizes, and more generally, genetic architectures, driven by both genetic ancestry composition of the subpopulation, and by behavioral, environmental and psychosocial exposures such as smoking, alcohol, nutrition, physical activity, and stress [14, 15], modifying the effect of genetic variants. While a handful of studies reported differences in genetic correlation across race/ethnic groups [16-18], these are limited in the number of studied phenotypes and race/ethnicities. Similar to race/ethnicity, while sex-specific heritabilities of complex phenotypes have been previously reported [19-21], sex-specific correlations between phenotypes have not been comprehensively studied.

The two main computational approaches that are used to estimate the genetic correlations are the genetic restricted maximum likelihood analysis (GREML)[22-24], which requires individual-level genotypes and is computationally challenging when analyzing datasets with thousands of individuals; and the linkage disequilibrium score regression (LDSC) [25], which uses genome-wide association study (GWAS) summary statistics. LDSC requires reliable GWASs, and can be inaccurate when there is genetic heterogeneity between the target sample and reference LD panel $[26,27]$ and thus cannot be 
medRxiv preprint doi: https://doi.org/10.1101/2021.09.05.21263126; this version posted September 10, 2021. The copyright holder for this preprint (which was not certified by peer review) is the author/funder, who has granted medRxiv a license to display the preprint in perpetuity.

It is made available under a CC-BY-NC-ND 4.0 International license.

used reliably for admixed or multi-ancestry analyses. Thus, both GREML and LDSC approaches are limited for datasets that include tens of thousands of genetically diverse individuals.

Here, we estimated the heritabilities, as well as genetic and environmental correlations between phenotypes in multi-ethnic as well as race/ethnicity- and sex-stratified analysis. First, we derived a closed form solution for the estimation of genetic and environmental correlation coefficients within the Haseman-Elston regression framework. Second, we applied the algorithm to study heritabilities and genetic correlations for 28 phenotypes in the Trans-Omics in Precision Medicine (TOPMed) program $[28,29]$ dataset, with large representation of White, Black and Hispanic/Latino populations. We then focused on the Hispanic/Latino population from the Hispanic Community Health Study / Study of Latinos (HCHS/SOL) cohort [30], and utilized available data on shared household (representing shared environmental exposure) and compared sex-specific genetic and environmental correlations across a larger panel of 61 phenotypes. Finally, we performed domain-level enrichment analysis to identify genetic and environmental correlations between phenotypic domains.

\section{Results}

\section{A Compendium of genetic correlations and heritabilities in the multi-ethnic TOPMed} dataset

We developed a novel closed-form estimator for genetic correlations between phenotypes within the Haseman-Elston regression framework. We studied the algorithm in simulations and compared the estimates to those estimated using GREML algorithm [4] implemented in the GCTA software [31] (Supplementary Fig 1), matching the latter for accuracy and improving the speed 100fold. We calculated phenotypic and genetic correlations between 28 phenotypes using 33,959 TOPMed individuals. Results are provided in Figure 1 and Supplementary Data file 1. There were 378 phenotype 
medRxiv preprint doi: https://doi.org/10.1101/2021.09.05.21263126; this version posted September 10, 2021. The copyright holder for this preprint (which was not certified by peer review) is the author/funder, who has granted medRxiv a license to display the preprint in perpetuity.

It is made available under a CC-BY-NC-ND 4.0 International license .

pairs in the dataset. Of these, $227(\sim 60 \%)$ had significant phenotypic correlations $R$, defined as p-value $<0.05$ and $|R|>0.05$ (Fig1A). Of these, 82 (36\%) also had genetic correlations $\rho_{k}$ with p-value $<0.05$ (Fig 1B, Fig 1D-inset). In contrast, out of the 151 phenotype pairs having $|R|<0.05$ and pvalue $<0.05,21$ pairs $(\sim 9 \%)$ had $\rho_{k}$ with $\mathrm{p}$-value $<0.05$ (for FDR corrected p-values see Supplementary

Data file 1).
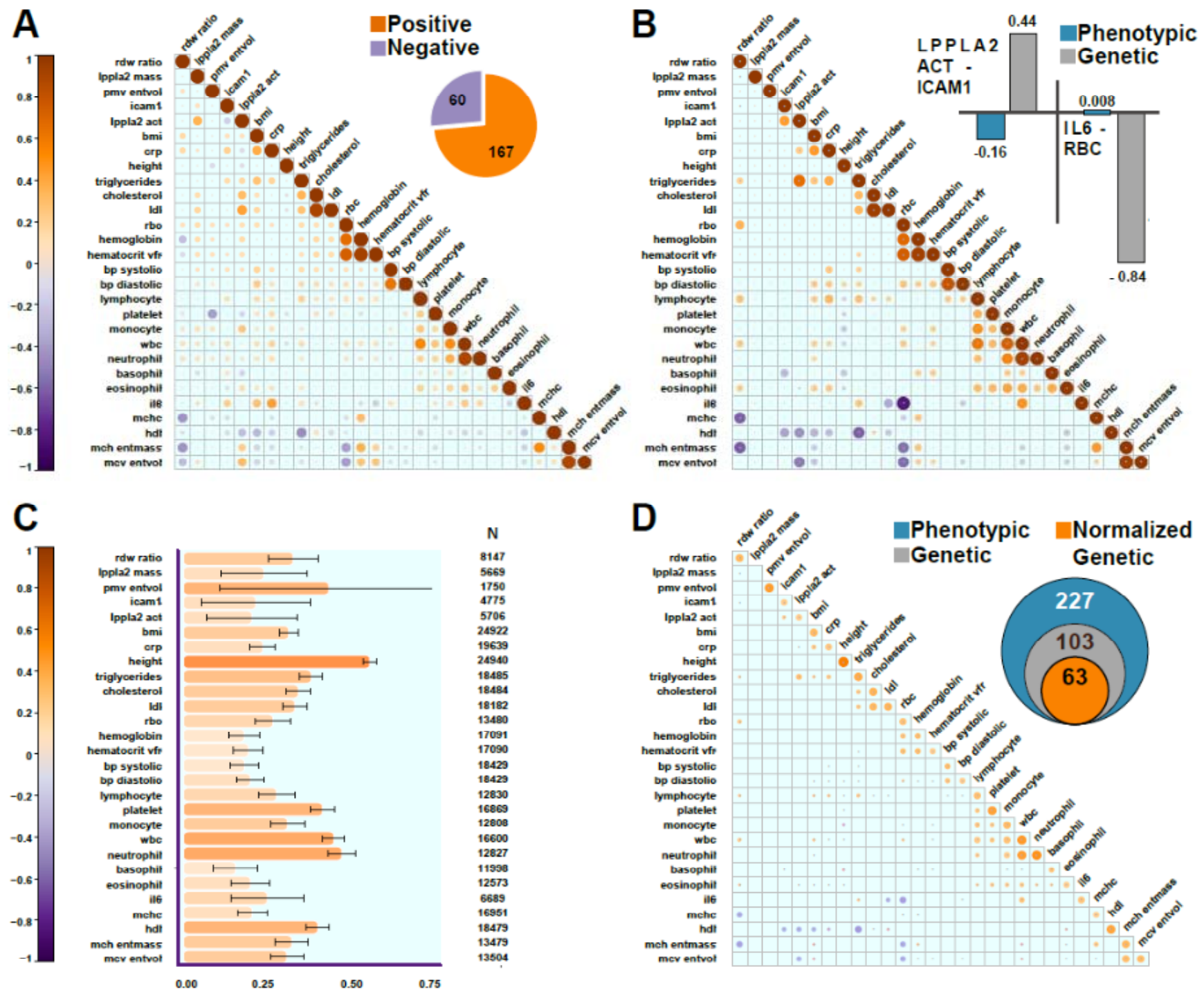

Figure 1. A third of observed phenotypic correlations between phenotypes in the combined TOPMed dataset have substantial genetic component. Correlation matrices where each column and row represent one of the 28 phenotypes in the TOPMed dataset and the intersection is the estimated correlation magnitude. Size and color of circle indicates the correlation strength; dark orange - positive, and dark purple - negative correlation. (A) Phenotypic correlations between the phenotypes. Inset - number of positive and negative correlated phenotype-pairs with p-value $<0.05$ and absolute phenotypic correlation above 0.05 (B) Estimated genetic correlations $\left(\rho_{k}\right)$ (shown only for phenotype pairs with p-value $<0.05$ between the phenotypes and $\left|\rho_{k}\right|>0.05$ ). Inset - examples of phenotypic and genetic correlation for two selected phenotypes where the absolute value of the genetic correlation is larger than that of the phenotypic correlation which complicates interpretability. (C) Estimated heritabilities for the studied phenotypes. (D) Normalized Genetic Correlations 
medRxiv preprint doi: https://doi.org/10.1101/2021.09.05.21263126; this version posted September 10, 2021. The copyright holder for this preprint (which was not certified by peer review) is the author/funder, who has granted medRxiv a license to display the preprint in perpetuity.

It is made available under a CC-BY-NC-ND 4.0 International license .

$\left(\rho_{N k}\right)$ between the phenotypes (shown only for phenotype pairs with p-value $<0.05$; and $\left|\rho_{N k}\right|>0.05$ ). Inset - number of phenotype-pairs with phenotype, $\rho_{k}$, and $\rho_{N k}$ with p-values $<0.05$ and absolute value of at least 0.05 in this dataset.

Our results agree well with previous reports. For example, we estimated a genetic correlation of $\rho_{k}=0.24$ between body-mass index (BMI) and triglycerides while Bulik-Sullivan et al. estimated it at 0.26[10] and Cadbi et al. at 0.2 [27]. Similarly, we estimated $\rho_{k}$ (genetic correlation) between Creactive protein and high-density lipoprotein (HDL) to be -0.24 , while Ligthart et al. reported it at -0.29 [32]. We also identified new and potentially clinically-relevant genetic correlations. For example, we estimated a significant $\rho_{k}=0.44$ between lipoprotein-associated phospholipase A2 and Intercellular adhesion molecule-1 which may important for the study of coronary artery disease biology; and multiple novel genetic correlations between white blood cell types and blood pressure, that are consistent with literature implicating a biological association between inflammation and hypertension [33, 34]; Supplementary Table 2).

We next estimated heritabilities for all the phenotypes in our multi-ethnic dataset (Fig 1C; Supplementary Table 3). The most highly heritable phenotypes are height (Fig 1C; 0.56) as well as multiple blood cell measurements such as neutrophil counts (Fig 1C; 0.47) and total white blood cell counts (WBC; Fig 1C; 0.45), similar to previous estimates [35, 36].

Normalized Genetic Correlation integrates genetic correlations with heritabilities to uncover the direct contribution of genetics to the observed phenotypic correlations

Our results identified multiple instances where the genetic correlation coefficient is larger than the phenotypic correlation (see Fig 1b inset for examples), emphasizing that the genetic correlation coefficient is not directly related to the phenotypic correlation [37, 38], and may be appreciable, even in pairs with overall very low phenotypic correlation. We thus introduce the concept of "normalized genetic correlation" $\left(\rho_{N k}\right)$, defined as the component of the observed phenotypic correlation $R$ 
explained by genetics in the decomposition $R=\hat{\rho}_{N k}+\hat{\rho}_{N \epsilon}$, where $\hat{\rho}_{N \epsilon}$ is the estimated normalized residual correlation.

We estimated $\rho_{N k}$ for all the pairs of phenotypes in our dataset (Fig 1D; Supplementary Table 4). Of phenotype pairs with genetic correlation with p-value $<0.05,61 \%$ had substantial $\widehat{\rho_{N k}}$, defined as $\left|\widehat{\rho_{N k}}\right|>0.05$, (Fig 1D-inset), corresponding to $23 \%$ of the phenotypic correlations with $\mathrm{R}>0.05$. For most of the phenotype-pairs, $\widehat{\rho_{N k}}$ is much lower than $\widehat{\rho_{k}}$ and is lower than the estimated $R$ (as expected). For example, for BMI and C-Reactive Proein (CRP), $\widehat{\rho_{k}}=0.45, \widehat{\rho_{N k}}=0.12$, and $\widehat{R}=0.38$. For HDL and triglycerides, $\widehat{\rho_{k}}=-0.61, \widehat{\rho_{N k}}=-0.24$, and $\hat{R}=-0.45$.

Genetic correlations between complex phenotypes and their heritabilities within race/ethnic groups

Out dataset includes 8,054 Black participants, 17,143 White participants and 8,762 Hispanics/Latinos (Supplementary Table 2). We estimated heritability, phenotypic, and genetic correlations within these race/ethnic groups and compared them to the multi-ethnic estimates (Figure 2; Supplementary Figure 2; Supplementary Data Files 1-4). 

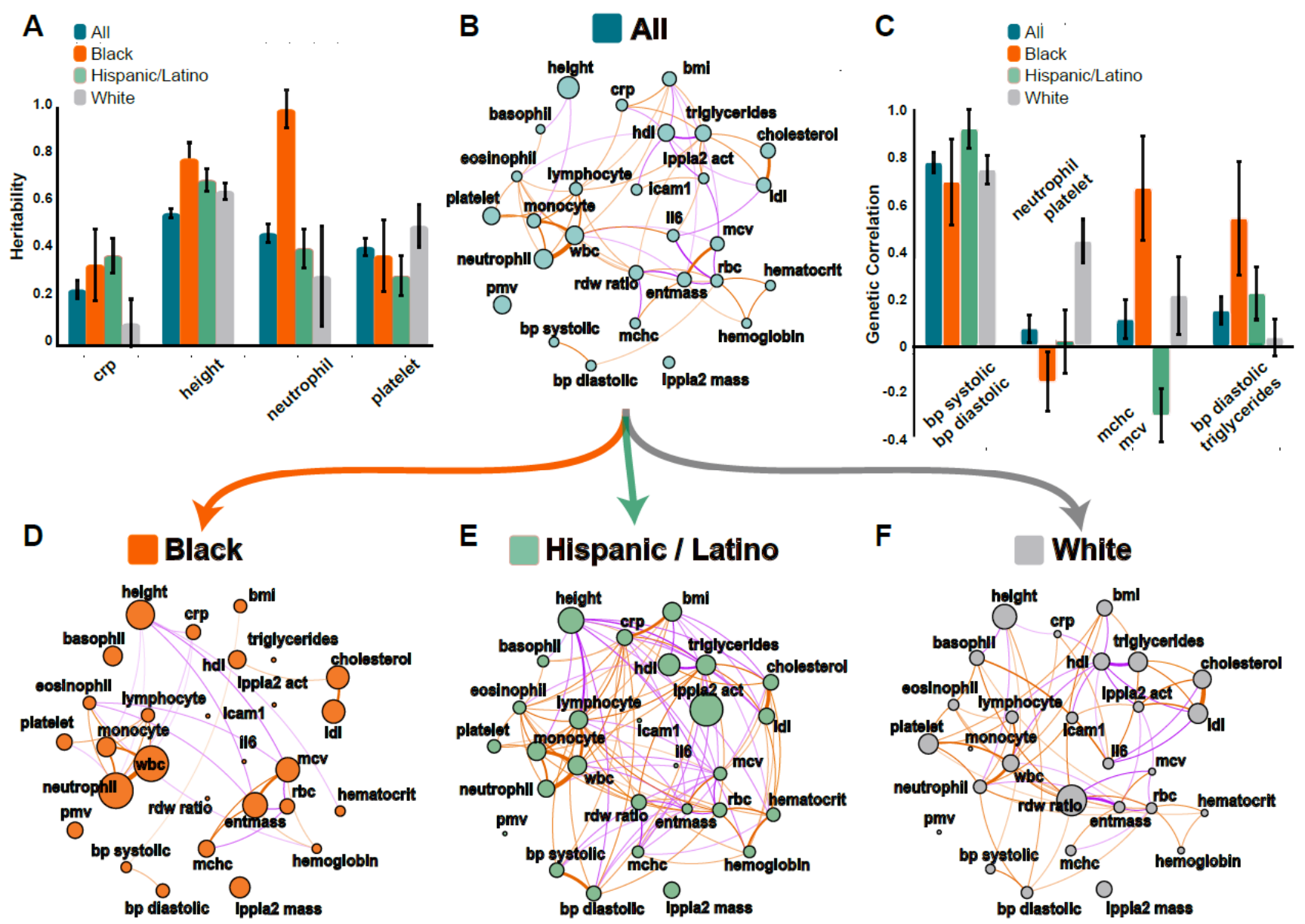

Figure 2. Some genetic correlations and heritabilities are race/ethnicity-specific. (B, D-F) Correlation plots where each phenotype is represented by a node and the correlations are represented by connections (edges) between nodes. The size of the node is proportional to the phenotype heritability. The thickness of the edge is proportional to the magnitude of correlation and the color represents direction: orange represents positive and purple negative correlation. (A) Examples of race/ethnic-specific heritabilities (C) Examples of race/ethnic-specific genetic correlations $\left(\rho_{k}\right)$ (B) Normalized genetic correlations $\left(\rho_{N k}\right)$ between the 28 phenotypes in the combined TOPMed dataset (p-value $\left.<0.05 ;\left|\rho_{N k}\right|>0.05\right)(\mathbf{D}, \mathbf{E}, \mathbf{F})$ Normalized genetic correlations $\left(\rho_{N k}\right)$ between the 28 phenotypes in the race/ethnic-specific subsets of the TOPMed dataset - Black (D, orange), Hispanic/Latino (E, marine) and White (F, grey).

While some phenotypes, such as HDL and eosinophil counts, have similar heritabilities across race/ethnicities (Fig 2A, D-F), many other heritabilities vary by race/ethnicity. For example, CRP is similarly heritable in the Blacks and Hispanics/Latinos $\left(\widehat{h_{2}}=0.34\right.$ and 0.38 respectively), but much less so in Whites $\left(\widehat{h_{2}}=0.09\right)$. In contrast, neutrophil counts are very heritable in Blacks $\left(\widehat{h_{2}}>\right.$ $0.99, S D=0.07)$, but are less so in Whites $\left(\widehat{h_{2}}=0.29\right)$ and Hispanics/Latinos $\left(\widehat{h_{2}}=0.41\right)$.

Similarly, multiple phenotype-pairs such as neutrophil and total WBC counts, and systolic and diastolic blood are similarly genetically correlated across race/ethnic groups (Fig 2C, D-F). However, 
medRxiv preprint doi: https://doi.org/10.1101/2021.09.05.21263126; this version posted September 10, 2021. The copyright holder for this preprint (which was not certified by peer review) is the author/funder, who has granted medRxiv a license to display the preprint in perpetuity.

It is made available under a CC-BY-NC-ND 4.0 International license .

many other $\rho_{k}$ differ by race/ethnicity (Fig2 C, D-F). For example, neutrophil and platelet counts have $\widehat{\rho_{k}}=0.44(\mathrm{p}$-value $<0.01)$ in Whites, but only $\widehat{\rho_{k}}=0.01$ in Hispanics/Latinos, and $\widehat{\rho_{k}}=-0.16(\mathrm{p}-$ value $<0.01$ ) in Blacks (Fig2 C, D-F). Similarly, diastolic blood pressure and triglycerides (Figure 2C) are strongly genetically correlated with $\widehat{\rho_{k}}=0.54$ in Blacks but have $\widehat{\rho_{k}}=0.03$ in Whites.

Overall, we detect 63 phenotype-pairs with $\left|\rho_{N k}\right|>0.05$ and p-value $<0.05$ in the combined multi-ethnic TOPMed dataset while detecting only 33 such pairs in the Black population, 105 in the Hispanic/Latino population and 69 in the White population. Supplementary Figure 2A visualizes the overlap between these correlations across race/ethnic groups (FDR corrected p-values are provided in Supplementary Data files).

Both genetics and shared-household drive phenotypic correlations in Hispanics/Latinos

We studied genetic correlations among a larger panel of 61 phenotypes in $n=7,678$ Hispanics/Latinos from the HCHS/SOL [30, 39]. The phenotypes represent 11 phenotypic domains: diabetes, cardiovascular disease, blood pressure, kidney function, lipids, lung function, sleep, anthropometrics, iron, RBC (red blood cells) and WBC (Fig 3, Supplementary Table 1). HCHS/SOL also has information about household sharing between participants, allowing for estimation of both genetic and environmental correlations between phenotypes. 

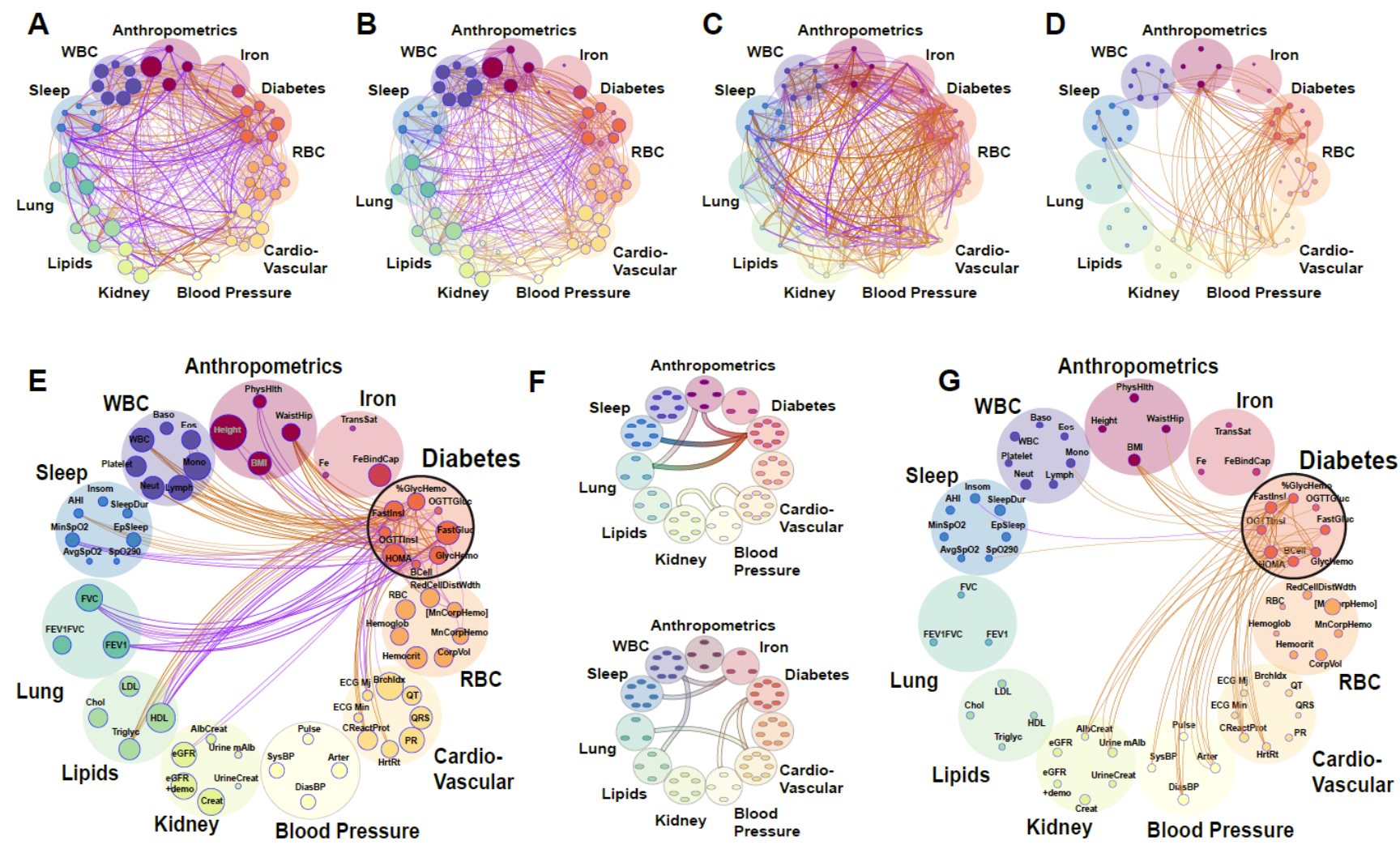

Figure 3. Genetics and shared Household factors contribute to associations between phenotypes in Hispanics/Latinos. (A-F) Correlation plots between the 61 phenotypes in the TOPMed HCHS/SOL dataset. Each phenotype is represented by a node (colored small circles) with the size of the circle proportional to the phenotype heritability. The correlations are represented by connections (edges) between nodes (phenotypes). The nodes are grouped into phenotypic domains (colored semi-transparent circles labelled Anthropometrics, Iron, etc.). The thickness of the edge is proportional to the strength of correlation and the color represents magnitude: orange represents positive and purple negative correlation. (A) Genetic correlations $\left(\rho_{k}\right)$ between the 61 phenotypes (p-value $\left.<0.05 ;\left|\rho_{k}\right|>0.05\right)$ (B) Normalized genetic correlations $\left(\rho_{N k}\right)$ between the 61 phenotypes (p-value $<0.05 ;\left|\rho_{N k}\right|>0.05$ ) (C) Household correlations (analog of genetic correlation but for household data; see Materials and Methods) between the 61 phenotypes (p-value $\left.<0.05 ;\left|\boldsymbol{\rho}_{H}\right|>0.05\right)$ (D) Normalized household correlations $\left(\rho_{N h}\right)$ between the 61 phenotypes (p-value $<0.05$; $\left.\left|\rho_{N h}\right|>0.05\right)$. (E+G) A focused look on the normalized genetic correlations for the contribution to the diabetes phenotypedomain of genetics $(\mathbf{E})$ and shared household $(\mathbf{G})$. Only the correlations for the diabetes phenotype-group are displayed. (F) Connections represent significantly enriched correlations between the phenotypic domains. (F-Top) represents genetic correlations and (F-bottom) Household correlations.

We estimated $\rho_{k}$ and $\rho_{N k}$ in conjunction with the equivalent household correlation measures $\rho_{h}$ and $\rho_{N h}$ for all the 1,830 pairs of phenotypes (figure 3 ). Out of the 1,830 phenotype-pairs, 1007 (or $\sim 55 \%$ ) have $|\hat{R}|>0.05$ (Supplementary Data file 5). Of these, 403 ( 40\%) also have $\left|\widehat{\rho_{k}}\right|>0.05$ (Fig 3A) and $412(\sim 41 \%)$ have $\left|\widehat{\rho_{h}}\right|>0.05$ with p-value $<0.05$. Of the genetically correlated pairs, 343 phenotype-pairs ( $34 \%$ of all phenotypic correlations) have $\left|\rho_{N k}\right|$ greater than 0.05. Of the 412 phenotype-pairs with significant $\widehat{\rho_{h}}$, only 91 are of $\left|\rho_{N h}\right|>0.05(\sim 9 \%$ of all phenotypic correlations). Unlike heritabilities, the estimated variance components for household are 
medRxiv preprint doi: https://doi.org/10.1101/2021.09.05.21263126; this version posted September 10, 2021. The copyright holder for this preprint (which was not certified by peer review) is the author/funder, who has granted medRxiv a license to display the preprint in perpetuity.

It is made available under a CC-BY-NC-ND 4.0 International license .

quite low for all phenotypes (Supplementary Data File 5), resulting in low $\rho_{N h}$ compared to $\rho_{N k}$. An interesting contrast between the genetic and household correlation is in the diabetes domain (Figure 3E, G). The diabetes domain has strong household correlations with blood pressure domain phenotypes (but no observed genetic correlations) and has strong genetic correlations with lung and lipid domain phenotypes (but no observed household correlations).

\section{Shared genetic and environmental basis between phenotypic domains via group enrichment analysis}

The genetic correlations are distributed non-uniformly with regard to the phenotypic domains. Domain enrichment analysis showed a strong enrichment of the number of intra-group correlations for all the 11 group-phenotypes (Supplementary Data File 5). Figure 3F visualizes the significant domainlevel correlations ( $\mathrm{p}$-value $<0.05$ ) estimated in enrichment analysis. Significant inter-group genetic correlations are estimated between the blood pressure domain and the kidney and cardiovascular domains; between the anthropometrics domain and the lung and diabetes domains; and between the diabetes domain and the anthropometrics, lung and sleep domains (Fig 3F-Top). Domain-level correlations due to shared household do not mirror the genetic ones (Fig 3F-Bottom vs Top). Specifically, we see that shared household affects the correlations between the diabetes and the bloodpressure and cardiovascular domains; the iron domain and the sleep and WBC domains; and the cardiovascular and lung domains (Fig 3F-Bottom).

Heritabilities as well as genetic and environmental correlations between complex human phenotypes differ across sexes

To obtain sufficient sample size, we expanded our analysis to include all genotyped participants in the HCHS/SOL cohort using imputed genetic data, for a total of 12,565 participants: 5,175 males and 7,390 females. Results are provided in Figure 4, with the number of significant correlations and 
medRxiv preprint doi: https://doi.org/10.1101/2021.09.05.21263126; this version posted September 10, 2021. The copyright holder for this preprint (which was not certified by peer review) is the author/funder, who has granted medRxiv a license to display the preprint in perpetuity.

It is made available under a CC-BY-NC-ND 4.0 International license .

overlaps between sexes visualized in Supplementary Figure 4. Supplementary Figure 5 and Supplementary Table 5 further provide additional results from sex-stratified genetic correlation analysis in TOPMed Whites. Overall, there were notable differences between both genetic and environmental correlations between the sexes. For example, the phenotype pair BMI and AHI (Apnea Hypopnea Index) had $\widehat{\rho_{k}}=0.88$ with $\mathrm{p}$-value $<0.01$ in men but $\widehat{\rho_{k}}=0.32$ with $\mathrm{p}$-value $=0.02$ in women; and WaistHip (waist to hip ratio) and GlycHemo (Glycosylated Hemoglobin in SI units) had $\widehat{\rho_{k}}=0.6$ with $\mathrm{p}$-value $<0.01$ in females but $\widehat{\rho_{k}}=0.35$ with $\mathrm{p}$-value $<0.01$ in males. Similarly, the household correlation for HOMA (HOMA-IR index of Insulin Resistance) and Height is $\widehat{\rho_{h}}=0.75$ with p-value $<0.01$ in men but $\widehat{\rho_{h}}=0.43$ with p-value $<0.01$ in women.
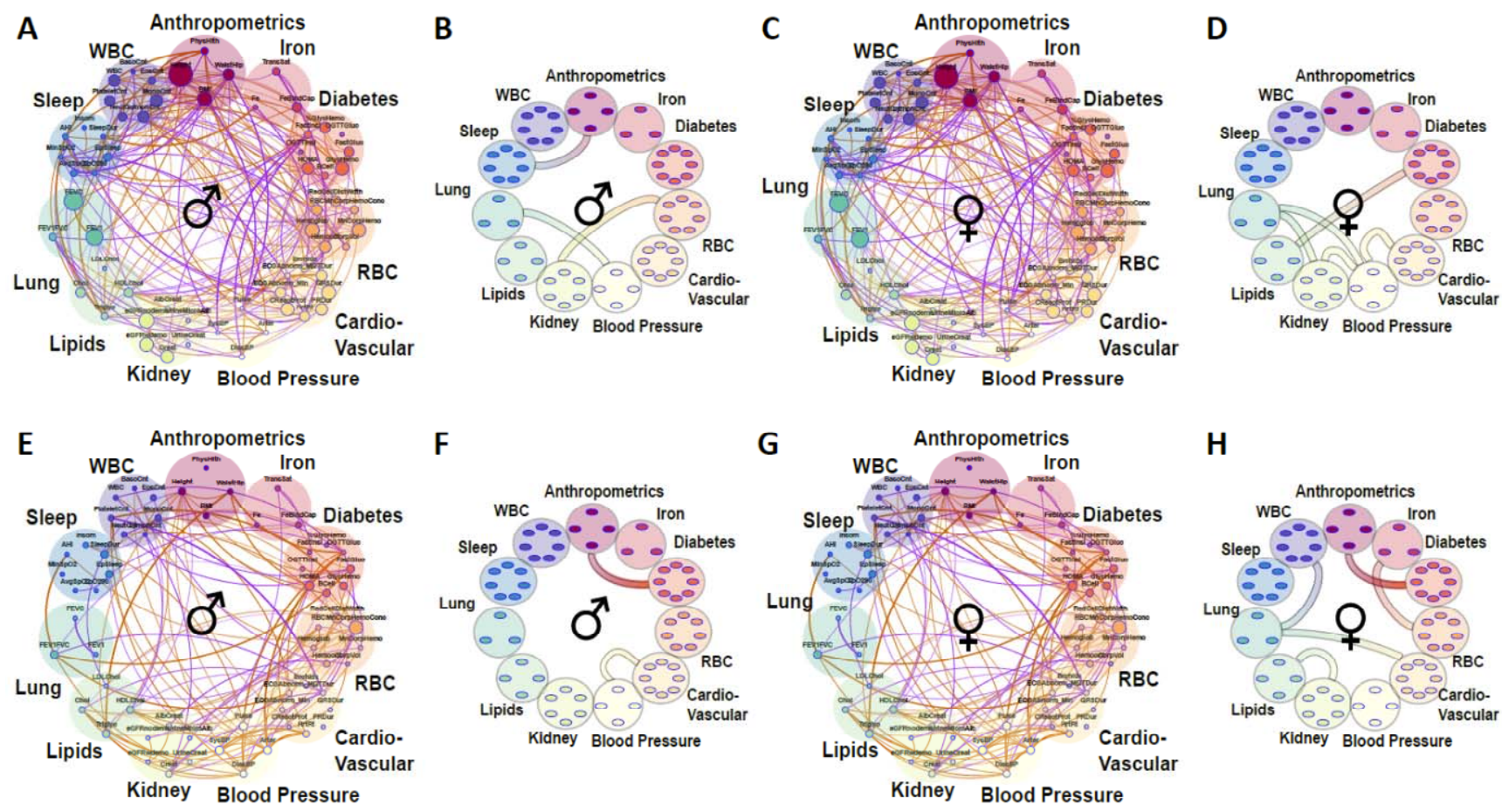

Figure 4. Sex differences in genetic and environmental correlations and heritabilities. (A, C, E, G) Correlation plots where each phenotype is represented by a node and the correlations are represented by connections (edges) between nodes. The size of the node is proportional to the phenotype heritability. The thickness of the edge is proportional to the strength of correlation and the color represents magnitude: orange represents positive and purple negative correlation. Shown are genetic correlations $\left(\rho_{k}\right)$ between the 61 phenotypes in the extended HCHS/SOL dataset (p-value $<0.05 ;\left|\rho_{k}\right|>0.05$ ). Correlations and heritabilities as measured in males $(\mathbf{A}, \mathbf{E})$ and females $(\mathbf{C}, \mathbf{G})$. (B, D, F, H) Significantly enriched correlations between the phenotypic domains. The top panels represent genetic correlations (A-D) and the bottom panels (EH) represent the environmental correlations. 
medRxiv preprint doi: https://doi.org/10.1101/2021.09.05.21263126; this version posted September 10, 2021. The copyright holder for this

preprint (which was not certified by peer review) is the author/funder, who has granted medRxiv a license to display the preprint in perpetuity.

It is made available under a CC-BY-NC-ND 4.0 International license .

Multiple differences can be observed at the domain level (Figure $4 \mathrm{~B}+\mathrm{D}, \mathrm{F}+\mathrm{H}$ ). For example, while the correlations between Kidney function and RBC (red blood cells) are predominantly genetic in males (Figure 4 B vs D), and do not reach significance threshold in females, the correlations between Anthropometrics and Diabetes are predominantly due to shared household regardless of sex (Figure $4 \mathrm{~F}$ vs $\mathrm{H})$. The same correlations can be differentially driven in sex-dependent manner as Blood Pressure interacts with Cardio-vascular domain through genetics in females but through environment in males (Figure 4 D vs F).

\section{Discussion}

We developed and implemented a novel, computationally efficient framework to estimate genetic and environmental correlations between phenotypes, and their contributions to the overall phenotypic correlation. We systematically interrogated heritabilities and genetic correlations between all phenotype-pairs of 28 blood pressure, lipids, blood counts, inflammation, and anthropometrics/demographic phenotypes from individual-level TOPMed dataset totaling 33,959 individuals, further stratified by race/ethnic group. We then focused on Hispanic/Latino participants from the HCHS/SOL cohort and estimated heritabilities and genetic and environmental (due to shared household) correlations between 61 phenotypes from 11 domains (diabetes, cardiovascular, blood pressure, kidney, lipids, lung, sleep, anthropometrics, iron, RBC, and WBC), further stratified by sex. Finally, we identified enriched genetic and environmental correlations at the domain level.

The genetic correlations for many of the phenotype pairs in our dataset have never been reported in the literature, and previous reports typically evaluated correlations in ancestrally uniform cohorts mostly without Black and Hispanic/Latino participants. The estimated heritabilities agree well with previously reported values from large population-based studies, and are slightly lower than heritability estimates from twin studies [5, 22, 24, 35, 40]. A recent report showed that higher 
heritability can be recovered from WGS studies by separating genetic variants into bins of allele frequencies, and constructing multiple genetic relationship matrices within those bins [41]. It is a topic of future research to study genetic correlations in these settings. Notably, our framework allows for multiple correlation matrices, enabling such investigation.

In our primary TOPMed dataset (as well as in several previous reports $[37,38]$ ), there are multiple instances where the estimated $\rho_{k}$ is greater than the phenotypic correlation $R$ (see Fig 1b inset). For example, while we estimated a modest phenotypic correlation of $\hat{R}=0.11$ between CRP and lymphocyte counts, the corresponding genetic correlation estimate was $\widehat{\rho_{k}}=0.33$. Thus, it is impossible to infer what fraction of the observed correlation is driven by genetics. We therefore introduce the concept of "normalized genetic correlation coefficient" $\left(\rho_{N k}\right)$ as the component of the observed phenotypic correlation explained by genetics. This concept allows for identification of phenotype-pairs where genetics is a large contributor to the observed correlation. Of phenotype pairs with genetic correlation $\left|\rho_{k}\right|>0.05$, only $\sim 17 \%$ of all phenotype-pairs have "substantial" normalized genetic correlations, defined here as $\left|\rho_{N k}\right|>0.05$ (Fig 1D-inset). For CRP and lymphocytes, $\widehat{\rho_{N k}}=$ 0.09 , suggesting that the phenotypic correlation between these phenotypes is mainly driven by genetics (0.09 out of 0.11 , which is $81 \%)$.

We were also able to estimate genetic and environmental correlations in understudied Hispanics/Latinos from the HCHS/SOL. The proportions of phenotype-pairs with significant phenotypic, genetic, and normalized genetic correlations, are overall similar to those in TOPMed, despite the expanded panel of phenotypes. We were also able to identify $\sim 22 \%$ of phenotype-pairs having $\left|\rho_{h}\right|>0.05$ with p-value $<0.05$, but only $\sim 4 \%$ had normalized household correlation $\left|\rho_{N h}\right|>$ 0.05, due to the overall low phenotypic variance explained by household environment. Shared household may contribute to the correlation between phenotypes by environmental causes of these phenotypes, e.g. nutrition, indoor pollutants, hygiene and other lifestyle habits, etc. We found that the 
medRxiv preprint doi: https://doi.org/10.1101/2021.09.05.21263126; this version posted September 10, 2021. The copyright holder for this

association of diabetes phenotypes with lipids and lung functions is largely genetic, while the diabetes phenotypes' associations with kidney function and blood pressure is mostly driven by shared environment. Future work is needed to study the implication of these results for treating complex cardiometabolic conditions.

In TOPMed, we studied heritabilities and genetic correlations stratified by self- or studyreported race/ethnicity. Some of the correlations and heritabilities differ across groups. This is not surprising given previous reports of differences in phenotypic distributions and disease prevalence across race/ethnicities [11-13]. For some phenotypes, the heritabilities were higher when estimated in each race/ethnic group separately. For example, while the estimated heritability for height in the combined dataset is $\widehat{h_{2}}=0.56$, it was $\widehat{h_{2}}=0.65$ in Whites (similar to previous reports of $\sim 0.6-0.62$ for individuals of European ancestry from TOPMed and UK Biobank [35]), $\widehat{h_{2}}=0.7$ for Hispanics/Latinos and $\widehat{h_{2}}=0.79$ for Blacks. Different heritability estimates between combined and stratified models are likely due to more precise modelling of variances in the stratified models.

Similarly, when studying genetic correlations, we found fewer phenotype pairs with significant normalized genetic correlations in the combined TOPMed dataset (63 pairs) than in either of the comprising White and Hispanic/Latino race/ethnic groups (69 and 105 accordingly), despite lower sample sizes, but not in Blacks (33 pairs), and the correlations do not entirely overlap. The results of the combined dataset in Figure 1 likely represent the "consensus" genetic relationships of the phenotype-pairs, e.g. the pairs that behave consistently in the majority of the constituent race/ethnic groups. Interestingly, the Black group had the lowest number of significant genetically-correlated phenotype-pairs which may be due to the high genetic variability of African genetic ancestry (which is substantial in U.S. admixed Blacks) combined with low sample size and thus lower power [41].

In the HCHS/SOL cohort, where 61 phenotypes were available, we performed domain-level enrichment analysis akin to pathway enrichment analysis in gene expression studies. We defined 
medRxiv preprint doi: https://doi.org/10.1101/2021.09.05.21263126; this version posted September $10,2021$. The copyright holder for this

domains as sets of phenotypes that capture similar underlying "latent" phenotypes, with the limitations that groups of phenotypes assigned for the same domain may still capture complex underlying biology, i.e. are not measures of exactly the same latent phenotype (e.g. insomnia and mean oxygen saturation during sleep, while can be correlated in individuals with obstructive sleep apnea, may also capture different pathophysiological disorders). While more study is needed, domain analysis should be less sensitive to individual variation in any particular phenotype. Interestingly, diabetes-domain correlations at the genetic level are enriched for sleep, anthropometry, and lipids domains, but at the environmental level, they are enriched for blood pressure and cardiovascular domains.

We performed sex-stratified analysis of genetic correlation in the Hispanics/Latinos, and found some sex differences. Overall, there were $32 \%$ phenotype-pairs with significant genetic correlations unique to females and $19 \%$ unique to males, and $23 \%$ and $15 \%$ with significant normalized genetic correlation. Similarly, there were $37 \%$ and $20 \%$ of pairs with significant household correlations in females and males accordingly but only $9 \%$ and $7 \%$ with significant normalized household correlation (Supplementary Figure $4 \mathrm{C}-\mathrm{H}$ ) which was also generally weaker. Multiple correlations between phenotypic domains were also different between the sexes.

In interpreting household correlations, we note that much like race/ethnicity in its relationship to genetic ancestry, gender is a social construct that is related to sex, but is heavily influenced by social practices and norms. Environmental correlations may differ between genders due to sociocultural differences between them. Here, we were not able to assess socio-cultural contributions related to gender roles to the household correlations, and we refer to "sex" rather than "gender", acknowledging that drivers of some of the estimated quantities are in fact gender, and not sex, based.

A specific strength of our study is the use of high-quality phenotypic and genotyping data from the diverse multi-ethnic TOPMed program. Further, all studies combined together are population-based cohort studies, reducing the likelihood of selection and other biases arising in studies 
medRxiv preprint doi: https://doi.org/10.1101/2021.09.05.21263126; this version posted September 10, 2021. The copyright holder for this preprint (which was not certified by peer review) is the author/funder, who has granted medRxiv a license to display the preprint in perpetuity.

It is made available under a CC-BY-NC-ND 4.0 International license .

following selected populations, such as case-control studies. Other strengths are the investigation of a large panel of phenotypes, evaluation of both genetic and environmental correlations, stratification by both race/ethnicity and sex, and the domain-level enrichment analysis. Nevertheless, this study also has a few limitations. For example, larger sample sizes, especially within stratified race/ethnic or sex groups, would enable stronger inferences. The use of self- or study-reported race/ethnicity rather than strata defined by genetic ancestry is also somewhat problematics. Still, we chose to proceed with these groupings, first, to reflect on currently used population groups in medical research, and second, because, given the use of Hispanics/Latinos who by definition, are admixed and cannot be represented by any detailed composition of genetic ancestry, there is no natural grouping that is based on genetic ancestry. As the field of genetic medicine grapples with the use of genetic ancestry and social definition of race/ethnicity [42], it would be important to re-consider models for genetic correlation analyses. Another limitation is imperfect definitions of phenotypic domains, that may not accurately capture underlying pathophysiology.

In summary, our work uncovered novel genetic and environmental correlations between phenotypes including differences by race/ethnicity and sex/gender. Future work includes the application of approaches from graph analysis field such as Gaussian Graphical Models [43, 44] [45] to discover directionality and causality; utilizing genetically correlated phenotypes to improve polygenic risk prediction models [46]; and studying genetic correlations by categories of genetic variants to capture the contributions of rare variants.

\section{Methods}

\subsection{Statistical models}

\section{Statistical model when genetic relatedness in the only modeled source of correlation}

Consider the linear model 


$$
y_{i}=x_{i}^{T} \beta+g_{i, 1} \alpha_{1}+\cdots+g_{i, d} \alpha_{d}+\varepsilon_{i}^{0}, \quad i=1, \ldots, n
$$

Model 1

in which the quantitative outcome $y_{i}$ is modelled by a regression on covariates $x_{i}$ and the additive effects of $d$ genetic variants $g_{1}, \ldots, g_{d}$; and $\varepsilon_{i}^{0} \sim \mathcal{N}\left(0, \sigma_{e}^{2}\right)$ are normally distributed errors across $n$ participants. Assuming that the genetic variants are independent random variables, each centered and scaled to have mean 0 and variance 1 , the mean and variance of $y_{i}$ are

$$
\begin{gathered}
E\left[y_{i}\right]=x_{i}^{T} \beta \\
\operatorname{var}\left(y_{i}\right)=\operatorname{var}\left(g_{1}\right) \times \alpha_{1}^{2}+\cdots+\operatorname{var}\left(g_{d}\right) \times \alpha_{d}^{2}+\sigma_{e}^{2}= \\
=\sum_{j=1}^{d} \alpha_{j}^{2}+\sigma_{e}^{2}=\sigma_{k}^{2}+\sigma_{e}^{2}
\end{gathered}
$$

Here, $\sigma_{k}^{2}, \sigma_{e}^{2}$ are the genetic and error variance components. Accordingly, narrow-sense heritability, which is the proportion of trait variance that is due to additive genetic factors is:

$$
\hat{h}^{2}=\frac{\sigma_{k}^{2}}{\sigma_{k}^{2}+\sigma_{e}^{2}}
$$

To model genetic correlation, we extend Model 1 into a two-trait model. For person $i$ :

$$
\begin{aligned}
& y_{i, 1}=x_{i}^{T} \beta_{1}+g_{i, 1} \alpha_{1,1}+\cdots+g_{i, d} \alpha_{1, d}+\epsilon_{i, 1}^{0} \\
& y_{i, 2}=x_{i}^{T} \beta_{2}+g_{i, 1} \alpha_{2,1}+\cdots+g_{i, d} \alpha_{2, d}+\epsilon_{i, 2}^{0}
\end{aligned}
$$

with error terms $\varepsilon_{i, 1}^{0}, \varepsilon_{i, 2}^{0}$ satisfying $\varepsilon_{i, l}^{0} \sim \mathcal{N}\left(0, \sigma_{\epsilon, l}^{2}\right), \operatorname{cor}\left(\epsilon_{i, 1}^{0}, \epsilon_{i, 2}^{0}\right)=\rho_{\epsilon}, \epsilon_{i, 1}^{0} \perp \epsilon_{j, 2}^{0}$ for $k \in\{1,2\}, i=$ $1, \ldots, n$.

Thus, the errors of the same person may be correlated for the two traits, but for different traits the error of person $i$ is independent of the error of person $j$. Consider the covariance between the two traits, again while making the simplifying assumption of independence between genetic variants:

$$
\begin{aligned}
\operatorname{cov}\left(y_{i, 1}, y_{i, 2}\right) & =\operatorname{cov}\left(x_{i}^{T} \beta_{1}+g_{i, 1} \alpha_{1,1}+\cdots+g_{i, d} \alpha_{1, d}+\epsilon_{i, 1}^{0}, x_{i}^{T} \beta_{2}+g_{i, 1} \alpha_{2,1}+\cdots+g_{i, d} \alpha_{2, d}+\epsilon_{i, 2}^{0}\right) \\
& =\operatorname{cov}\left(g_{i, 1} \alpha_{1,1}+\cdots+g_{i, d} \alpha_{1, d}+\epsilon_{i, 1}^{0}, g_{i, 1} \alpha_{2,1}+\cdots+g_{i, d} \alpha_{2, d}+\epsilon_{i, 2}^{0}\right)
\end{aligned}
$$




$$
=\sum_{k=1}^{d} \alpha_{1, k} \alpha_{2, k}+\sigma_{1} \sigma_{2} \rho_{e}=\sigma_{g_{1}} \sigma_{g_{2}} \rho_{k}+\sigma_{\epsilon, 1} \sigma_{\epsilon, 2} \rho_{e}
$$

where $\rho_{k}$ is the correlation between the genetic effect of the $k$-th variant on the two outcomes, and $\rho_{e}$ is the correlation between the residual errors of the two outcomes. Note that the transition to using $\rho_{k}$ at the final step treats the vectors of causal genetic effects $\alpha_{1}=\left(\alpha_{1,1} \ldots \alpha_{1, d}\right)^{T}, \alpha_{2}=\left(\alpha_{2,1} \ldots \alpha_{2, d}\right)^{T}$ as random variables with mean 0 , i.e., $E\left[\alpha_{i, j}\right]=0, \operatorname{var}\left(\alpha_{i, j}\right)=\sigma_{g, i}^{2}, i \in\{1,2\}, j=1, \ldots, d$.

Noting that for individuals $i, l, \operatorname{cov}\left(g_{i, j}, g_{l, j}\right)=k_{i, l}$, the probability of the two individuals sharing the same allele identically-by-decent [47, 48], an equivalent formulation supposes that the genetic effects can be modelled via $\mathbf{K}$, the $n \times n$ kinship matrix, tabulating the measure of genetic relationship between the $i$ and $j$ participants in its $i, j$ entry. Consider the vector form of the model for the $l$ outcome with correlated errors of trait $l=1,2$ :

$$
\begin{aligned}
y_{l} & =X \beta_{l}+\epsilon_{l}, \quad l=1,2 \\
\operatorname{cov}\left(\epsilon_{l}\right) & =\sigma_{\epsilon, l}^{2} \boldsymbol{I}_{n \times n}+\sigma_{k, l}^{2} \boldsymbol{K} \\
\operatorname{cov}\left(\epsilon_{1}, \epsilon_{2}\right) & =\sigma_{\epsilon, 1} \sigma_{\epsilon, 2} \rho_{\epsilon} \boldsymbol{I}_{n \times n}+\sigma_{k, 1} \sigma_{k, 2} \rho_{k} \boldsymbol{K}
\end{aligned}
$$

Model 2

Now the genetic correlation can be estimated using mixed model with two traits. However, this is computationally demanding, especially for very large data sets. Recently, [49] discussed the HasemanElston regression for variance components estimation, and demonstrated that the genetic variance components estimator corresponding to the kinship matrix, if it is independent of all other correlation matrices in a model with potentially multiple sources of correlation (which holds here, because we only have a single correlation matrix, the kinship matrix) are given by $\hat{\sigma}_{\epsilon, l}^{2}=\frac{\hat{\epsilon}_{l}^{T} \boldsymbol{K}^{-} \hat{\epsilon}_{l}}{\operatorname{tr}\left(\boldsymbol{K}^{-} \boldsymbol{K}^{-}\right)}$, where $\boldsymbol{K}^{-}$is the kinship matrix with all diagonal values set to zero. 


\section{An estimator of genetic correlation between two phenotypes}

We extend the Haseman-Elston approach for modelling the genetic correlations between two phenotypes. For the errors of persons $i$ and $j$, and phenotypes 1 and 2, under Model 2 we get:

$$
\begin{aligned}
& E\left[\epsilon_{1, i}, \epsilon_{2, i}\right]=\sigma_{\epsilon, 1} \sigma_{\epsilon, 2} \rho_{\epsilon}+\sigma_{k, 1} \sigma_{k, 2} \rho_{k} \\
& E\left[\epsilon_{1, i}, \epsilon_{2, j}\right]=\sigma_{k, 1} \sigma_{k, 2} \rho_{k} k_{i, j}
\end{aligned}
$$

Suppose for now that $\sigma_{k, 1}=\left|\sqrt{\sigma_{k, 1}^{2}}\right|$ and $\sigma_{k, 2}=\left|\sqrt{\sigma_{k, 2}^{2}}\right|$ are known. For estimating the genetic correlation between the two phenotypes, we take all pairs $\hat{\epsilon}_{1, i} \hat{\epsilon}_{2, j}$ of residuals (estimating the error terms) after regression on mean- model covariates for $i \neq j$, and regress them against the "covariate" $\sigma_{k, 1} \sigma_{k, 2} k_{i, j}$. From properties of linear regression, we get:

$$
\hat{\rho}_{k}=\frac{\sum_{i=1}^{n} \sigma_{k, 1} \sigma_{k, 2} k_{i, j} \hat{\epsilon}_{1, i} \hat{\epsilon}_{2, j}}{\sum_{i=1}^{n} \sigma_{k, 1}^{2} \sigma_{k, 2}^{2} k_{i, j}^{2}}=\frac{\sum_{i=1}^{n} k_{i, j} \hat{\epsilon}_{1, i} \hat{\epsilon}_{2, j}}{\sigma_{k, 1} \sigma_{k, 2} \sum_{i=1}^{n} k_{i, j}^{2}}=\frac{\hat{\epsilon}_{1}^{T} \boldsymbol{K}^{-} \hat{\epsilon}_{2}}{\sigma_{k, 1} \sigma_{k, 2} \operatorname{tr}\left(\boldsymbol{K}^{-} \boldsymbol{K}^{-}\right)}
$$

Now we can plug-in the estimators of $\sigma_{k, 1}, \sigma_{k, 2}$ to get:

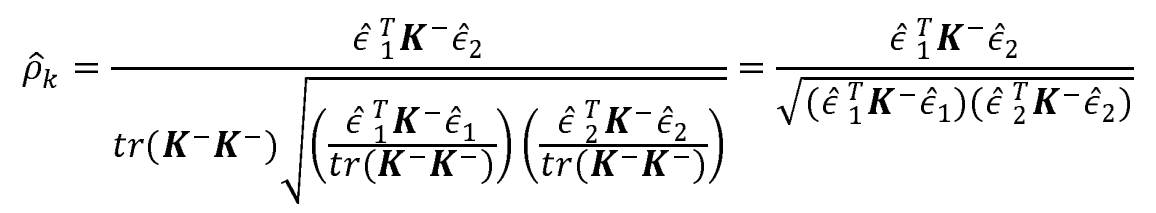

This estimator resembles that of the Pearson correlation parameter between the variables $\hat{\epsilon}_{1}$ and $\hat{\epsilon}_{2}$, as can be seen if one replaces the matrix $\boldsymbol{K}^{-}$by the identify matrix $\boldsymbol{I}$. Interestingly, this estimator does not involve the unknown variance parameters. It does include the estimated kinship parameters, which are treated as fixed.

\section{Extension to multiple correlation matrices and generalization}

We can use multiple relatedness matrices $(A, \ldots, K)$ with elements $\left(a_{i, j} . k_{i, j}\right)$ indicating the measure of relatedness between the $i$-th and $j$-th participants in its $i, j$ entry to model the variance. We can then estimate the variance components obtained from expressions of the form 


$$
E\left[\epsilon_{i}, \epsilon_{j}\right]=\sigma_{\epsilon}^{2} \times 1_{(i=j)}+\sigma_{a}^{2} a_{i, j}+\cdots+\sigma_{k}^{2} k_{i, j}
$$

(corresponding to Model 2 above) via a residual regression, i.e. by taking the vector all pairs of residuals $\hat{\epsilon}_{1, i} \hat{\epsilon}_{2, j}$ for all $i, j$. The HE design matrix, now re-defined (compared to [49]) to include rows corresponding to $\hat{\epsilon}_{1, i} \hat{\epsilon}_{2, j}$ with $i=j$, is given by:

$$
X_{\sigma}=\left(\begin{array}{cccc}
1 & a_{1,1} & \ldots & k_{1,1} \\
0 & a_{1,2} & \ldots & k_{1,2} \\
\vdots & \vdots & \ldots & \vdots \\
0 & a_{1, n} & \ldots & k_{1, n} \\
0 & a_{2,1} & \ldots & k_{2,1} \\
1 & a_{2,2} & \ldots & k_{2,2} \\
\vdots & \vdots & \ldots & \vdots \\
0 & a_{2, n} & \ldots & k_{2, n} \\
\vdots & \vdots & \ldots & \vdots \\
1 & a_{n, n} & \ldots & k_{n, n}
\end{array}\right)
$$

Similarly, the design matrix for estimating genetic correlation, obtained from expression of the form

$$
E\left[\epsilon_{1, i}, \epsilon_{2, j}\right]=\sigma_{\epsilon, 1} \sigma_{\epsilon, 2} \rho_{\epsilon} 1_{i=j}+\sigma_{a, 1} \sigma_{a, 2} \rho_{a} a_{i, j}+\cdots+\sigma_{k, 1} \sigma_{k, 2} \rho_{k} k_{i, j}
$$

Can be written (if the variance parameters were known) as:

$$
X_{\rho}=\left(\begin{array}{cccc}
\sigma_{\epsilon, 1} \sigma_{\epsilon, 2} & \sigma_{a, 1} \sigma_{a, 2} a_{1,1} & \ldots & \sigma_{k, 1} \sigma_{k, 2} k_{1,1} \\
0 & \sigma_{a, 1} \sigma_{a, 2} a_{1,2} & \ldots & \sigma_{k, 1} \sigma_{k, 2} k_{1,2} \\
\vdots & \vdots & \ldots & \vdots \\
0 & \sigma_{a, 1} \sigma_{a, 2} a_{1, n} & \ldots & \sigma_{k, 1} \sigma_{k, 2} k_{1, n} \\
0 & \sigma_{a, 1} \sigma_{a, 2} a_{2,1} & \ldots & \sigma_{k, 1} \sigma_{k, 2} k_{2,1} \\
\sigma_{\epsilon, 1} \sigma_{\epsilon, 2} & \sigma_{a, 1} \sigma_{a, 2} a_{2,2} & \ldots & \sigma_{k, 1} \sigma_{k, 2} k_{2,2} \\
\vdots & \vdots & \ldots & \vdots \\
0 & \sigma_{a, 1} \sigma_{a, 2} a_{2, n} & \ldots & \sigma_{k, 1} \sigma_{k, 2} k_{2, n} \\
\vdots & \vdots & \ldots & \vdots \\
\sigma_{\epsilon, 1} \sigma_{\epsilon, 2} & \sigma_{a, 1} \sigma_{a, 2} a_{n, n} & \ldots & \sigma_{k, 1} \sigma_{k, 2} k_{n, n}
\end{array}\right)
$$

Noting that:

$$
X_{\rho}=X_{\sigma}\left(\begin{array}{cccc}
\sigma_{\epsilon, 1} \sigma_{\epsilon, 2} & 0 & \ldots & 0 \\
0 & \sigma_{b, 1} \sigma_{b, 2} & \ldots & 0 \\
\vdots & \vdots & \ddots & \vdots \\
0 & 0 & \ldots & \sigma_{k, 1} \sigma_{k, 2}
\end{array}\right)=X_{\sigma} D_{\sigma}
$$

We get that: 


$$
\begin{gathered}
X_{\rho}^{T} X_{\rho}=D_{\sigma} X_{\sigma}^{T} X_{\sigma} D_{\sigma} \\
\left(X_{\rho}^{T} X_{\rho}\right)^{-1}=D_{\sigma}^{-1}\left(X_{\sigma}^{T} X_{\sigma}\right)^{-1} D_{\sigma}^{-1}
\end{gathered}
$$

Eq. 17

We also note the outcome matrices for estimating variance components and correlation parameters are:

$$
Y_{\sigma, 1}=\left(\begin{array}{c}
\epsilon_{1,1} \epsilon_{1,1} \\
\epsilon_{1,1} \epsilon_{1,2} \\
\vdots \\
\epsilon_{1,1} \epsilon_{1, n} \\
\epsilon_{1,2} \epsilon_{1,1} \\
\epsilon_{1,2} \epsilon_{1,2} \\
\vdots \\
\epsilon_{1,2} \epsilon_{1, n} \\
\vdots \\
\epsilon_{1, n} \epsilon_{1, n}
\end{array}\right), Y_{\sigma, 2}=\left(\begin{array}{c}
\epsilon_{2,1} \epsilon_{2,1} \\
\epsilon_{2,1} \epsilon_{2,2} \\
\vdots \\
\epsilon_{2,1} \epsilon_{2, n} \\
\epsilon_{2,2} \epsilon_{2,1} \\
\epsilon_{2,2} \epsilon_{2,2} \\
\vdots \\
\epsilon_{2,2} \epsilon_{2, n} \\
\vdots \\
\epsilon_{2, n} \epsilon_{2, n}
\end{array}\right), Y_{\rho}=\left(\begin{array}{c}
\epsilon_{1,1} \epsilon_{2,1} \\
\epsilon_{1,1} \epsilon_{2,2} \\
\vdots \\
\epsilon_{1,1} \epsilon_{2, n} \\
\epsilon_{1,2} \epsilon_{2,1} \\
\epsilon_{1,2} \epsilon_{2,2} \\
\vdots \\
\epsilon_{1,2} \epsilon_{2, n} \\
\vdots \\
\epsilon_{1, n} \epsilon_{2, n}
\end{array}\right)
$$

Eq. 18

Therefore:

$$
\begin{array}{r}
\left(\begin{array}{c}
\sigma_{\epsilon, 1}^{2} \\
\sigma_{a, 1}^{2} \\
\vdots \\
\sigma_{k, 1}^{2}
\end{array}\right)=\left(X_{\sigma}^{T} X_{\sigma}\right)^{-1} X_{\sigma}^{T} Y_{\sigma, 1},\left(\begin{array}{c}
\sigma_{\epsilon, 2}^{2} \\
\sigma_{a, 2}^{2} \\
\vdots \\
\sigma_{k, 2}^{2}
\end{array}\right) \\
=\left(X_{\sigma}^{T} X_{\sigma}\right)^{-1} X_{\sigma}^{T} Y_{\sigma, 2}
\end{array}
$$

and:

$$
\begin{aligned}
\left(\begin{array}{c}
\sigma_{\epsilon, 1}^{2} \\
\sigma_{a, 1}^{2} \\
\vdots \\
\sigma_{k, 1}^{2}
\end{array}\right) & =\left(X_{\rho}^{T} X_{\rho}\right)^{-1} X_{\rho}^{T} Y_{\rho} \\
& =D_{\sigma}^{-1}\left(X_{\sigma}^{T} X_{\sigma}\right)^{-1} D_{\sigma}^{-1} D_{\sigma} X_{\sigma}^{T} Y_{\rho} \\
& =D_{\sigma}^{-1}\left(X_{\sigma}^{T} X_{\sigma}\right)^{-1} X_{\sigma}^{T} Y_{\rho}
\end{aligned}
$$

Because the $l_{\text {th }}$ entry of $D_{\sigma}$ is $\sigma_{1, l} \sigma_{2, l}$ we have then for $\rho_{l}$ :

$$
\begin{aligned}
\rho_{l} & =\frac{\left[\left(X_{\sigma}^{T} X_{\sigma}\right)^{-1} X_{\sigma}^{T} Y_{\rho}\right]_{l}}{\sigma_{1, l} \sigma_{2, l}} \\
& =\frac{\left[\left(X_{\sigma}^{T} X_{\sigma}\right)^{-1} X_{\sigma}^{T} Y_{\rho}\right]_{l}}{\sqrt{\left[\left(X_{\sigma}^{T} X_{\sigma}\right)^{-1} X_{\sigma}^{T} Y_{\sigma, 1}\right]_{l}\left[\left(X_{\sigma}^{T} X_{\sigma}\right)^{-1} X_{\sigma}^{T} Y_{\sigma, 2}\right]}}
\end{aligned}
$$


To prove that this is a generalized Pearson correlation, we only need to show that $\left[\left(X_{\sigma}^{T} X_{\sigma}\right)^{-1} X_{\sigma}^{T} Y_{\rho}\right]_{l}$ is a bilinear form, with the matrix completely defined by the $l_{\text {th }}$ row of $\left(X_{\sigma}^{T} X_{\sigma}\right)^{-1} X_{\sigma}^{T}$. This is simple to see, because the entries of $X_{\sigma}^{T} Y_{\rho}$ are:

$$
X_{\sigma}^{T} Y_{\rho}=\left(\begin{array}{c}
\epsilon_{1}^{T} \boldsymbol{I} \epsilon_{2} \\
\epsilon_{1}^{T} \boldsymbol{A} \epsilon_{2} \\
\vdots \\
\epsilon_{1}^{T} \boldsymbol{K} \epsilon_{2}
\end{array}\right)
$$

Similarly:

$$
X_{\sigma}^{T} Y_{\sigma, 1}=\left(\begin{array}{c}
\epsilon_{1}^{T} \boldsymbol{I} \epsilon_{1} \\
\epsilon_{1}^{T} \boldsymbol{A} \epsilon_{1} \\
\vdots \\
\epsilon_{1}^{T} \boldsymbol{K} \epsilon_{1}
\end{array}\right), X_{\sigma}^{T} Y_{\sigma, 2}=\left(\begin{array}{c}
\epsilon_{2}^{T} \boldsymbol{I} \epsilon_{2} \\
\epsilon_{2}^{T} \boldsymbol{A} \epsilon_{2} \\
\vdots \\
\epsilon_{2}^{T} \boldsymbol{K} \epsilon_{2}
\end{array}\right)
$$

and the $l_{\text {th }}$ row of $\left(X_{\sigma}^{T} X_{\sigma}\right)^{-1}$ determines the weights in the following expression:

Thus for

$$
\left[\left(X_{\sigma}^{T} X_{\sigma}\right)^{-1} X_{\sigma}^{T} Y_{\rho}\right]_{l}=\epsilon_{1}^{T}\left[\omega_{\epsilon, l} \boldsymbol{I}+\omega_{a, l} \boldsymbol{A}+\cdots+\omega_{k, l} \boldsymbol{K}\right] \epsilon_{1}
$$

$$
\boldsymbol{S}_{\boldsymbol{l}}=\omega_{\epsilon, l} \boldsymbol{I}+\omega_{a, l} \boldsymbol{A}+\cdots+\omega_{k, l} \boldsymbol{K}
$$

We get

$$
\hat{\rho}_{l}=\frac{\hat{\epsilon}_{1}^{T} \boldsymbol{S}_{l} \hat{\epsilon}_{2}}{\sqrt{\left(\hat{\epsilon}_{1}^{T} \boldsymbol{S}_{l} \hat{\epsilon}_{1}\right)\left(\hat{\epsilon}_{2}^{T} \boldsymbol{S}_{l} \hat{\epsilon}_{2}\right)}}
$$

\section{Deriving confidence intervals for estimated correlation coefficients}

We propose two methods to compute confidence intervals for the estimated correlation coefficients. First, using the Fisher's transformation, which was developed to estimate confidence intervals for the standard Pearson correlation coefficient, and second, using block bootstrap.

\section{Confidence intervals using the Fisher's transform}

Fisher's transformation converts the distribution of the correlation coefficients to a normal one and thus allows to calculate confidence intervals (and corresponding p-values) for the correlation coefficient 
using the values of the correlation coefficient and the sample size $[50,51]$. Since we show that calculating genetic correlation is equal to calculating standard correlation for adjusted phenotypes (Eq. 29), the Fisher method is equally applicable for genetic correlation coefficient with the modification of plugging-in "effective sample size" to account for the modelled correlation structure between the two traits. Specifically, Fisher's z-transformation of a correlation coefficient $\rho$ is defined as:

$$
z=\frac{1}{2} \ln \left(\frac{1+\rho}{1-\rho}\right)=\operatorname{arctanh}(\rho)
$$

If the two variables for which the correlation is measured have a bivariate normal distribution and are independent and identically distributed, then $z$ is approximately normally distributed with mean $\mu$ and standard error $\sigma$ given by:

$$
\begin{gathered}
\mu=\frac{1}{2} \ln \left(\frac{1+\rho}{1-\rho}\right) \\
\sigma=\frac{1}{\sqrt{N_{e f f}-3}}
\end{gathered}
$$

and $N_{\text {eff }}$ being the effective sample size of our sample equal to:

$$
N_{e f f}=\sqrt{\operatorname{tr}\left(S_{l} S_{l}^{T}\right)}
$$

where $\boldsymbol{S}_{\boldsymbol{l}}$ is the weighted matrix described in $E q .28$. The coverage of this approach was verified in simulations via comparisons to the block Bootstrap method described below (see Supplementary Fig 1).

\section{Confidence intervals using block Bootstrap}

Multiple participants in our datasets are genetically related (and thus correlated), which violates the assumption of the standard bootstrap method. We thus performed the block bootstrap procedure to derive the confidence intervals and p-values as described in [52]. Briefly, related individuals were grouped into blocks (via $3^{\text {rd }}$ degree kinship) and the sampling procedure was at the level of blocks. 
Standard deviations and confidence intervals were calculated from the bootstrapped values that were Fisher's transformed.

\subsection{Heritability-Normalized Genetic Correlation}

For simplicity, focus on the single correlation matrix settings (the derivation here naturally extends to multiple sources of correlation). Recall Model 2 - the phenotypic correlation coefficient $\boldsymbol{R}$ is equal to:

$$
\begin{gathered}
R=\frac{\operatorname{cov}\left(\epsilon_{1}, \epsilon_{2}\right)}{\sqrt{\operatorname{cov}\left(\epsilon_{1}\right) \operatorname{cov}\left(\epsilon_{2}\right)}} \\
=\left(\frac{\sigma_{\epsilon, 1} \sigma_{\epsilon, 2}}{\sqrt{\sigma_{\epsilon, 1}^{2}+\sigma_{k, 1}^{2}} \sqrt{\sigma_{\epsilon, 2}^{2}+\sigma_{k, 2}^{2}}} \times \rho_{\epsilon}\right)+\left(\frac{\sigma_{k, 1} \sigma_{k, 2}}{\sqrt{\sigma_{\epsilon, 1}^{2}+\sigma_{k, 1}^{2}} \sqrt{\sigma_{\epsilon, 2}^{2}+\sigma_{k, 2}^{2}}} \times \rho_{k}\right)
\end{gathered}
$$

We then define Normalized Genetic Correlation $\left(\rho_{N k}\right)$ as

$$
\rho_{N k}=\left(\frac{\sigma_{k, 1} \sigma_{k, 2}}{\sqrt{\sigma_{\epsilon, 1}^{2}+\sigma_{k, 1}^{2}} \sqrt{\sigma_{\epsilon, 2}^{2}+\sigma_{k, 2}^{2}}} \times \rho_{k}\right)
$$

and Normalized Residual Correlation as $\rho_{N \epsilon}=\frac{\sigma_{\epsilon, 1} \sigma_{\epsilon, 2}}{\sqrt{\sigma_{\epsilon, 1}^{2}+\sigma_{k, 1}^{2}} \sqrt{\sigma_{\epsilon, 2}^{2}+\sigma_{k, 2}^{2}}} \times \rho_{\epsilon}$.

This is a natural decomposition of phenotypic correlation into two components:

$$
R=\rho_{N k}+\rho_{N \epsilon}
$$

Unlike standard genetic correlation, $\rho_{N k}$ is the genetic correlation adjusted (normalized) for both traits' heritabilities and variances and crucially it represents a fraction of the phenotypic correlation, normalized for the total phenotypic correlation, that is due to genetics. Thus, the normalized correlation terms are never larger than the phenotypic correlation $R$, because they sum to $R$.

\subsection{Simulation studies}

We studied the accuracy of the proposed method for estimating genetic correlations and for calculating confidence intervals in simulations. We used correlation matrices from the HCHS/SOL representing kinship and shared household to generate realistic correlation structures. In all simulations, data were 
generated by first sampling two uncorrelated error vectors $\left(\epsilon_{1}, \epsilon_{2}\right)$ from a standard normal distribution. We next simulated the covariance structure according to our model:

$$
\begin{aligned}
\operatorname{cov}\left(\epsilon_{l}\right) & =\sigma_{\epsilon, l}^{2} \boldsymbol{I}_{n \times n}+\sigma_{k, l}^{2} \boldsymbol{K}+\sigma_{h, l}^{2} \boldsymbol{H} \\
\operatorname{cov}\left(\epsilon_{1}, \epsilon_{2}\right) & =\sigma_{\epsilon, 1} \sigma_{\epsilon, 2} \rho_{\epsilon} \boldsymbol{I}_{n \times n}+\sigma_{k, 1} \sigma_{k, 2} \rho_{k} \boldsymbol{K}+\sigma_{h, 1} \sigma_{h, 2} \rho_{h} \boldsymbol{H}
\end{aligned}
$$

The matrix $\mathbf{K}$ represents kinship, and $\mathbf{H}$ represents shared household. All simulations were performed 1,000 times with different sample sizes $(1000,4000$, and 7706 , the latter is sample size of HCHS/SOL individuals in TOPMed freeze 8 which is the smallest subpopulation in this study) and values of $\rho_{k}$ and $\rho_{h}$ ranging from 0 to 1 in increments of 0.1 . The variance components reported here were set to typical values for phenotypes from our dataset and equal to $\sigma=\left(\sigma_{k, 1} \sigma_{k, 2}, \sigma_{h, 1}, \sigma_{h, 2}\right)=(0.6,0.7,0.4,0.3)$ corresponding to e.g., HDL, Height, Fasting Glucose levels and Eosinophil counts. The confidence intervals and p-values were calculated via the Fisher transformation and block bootstrap methods as described above.

\subsection{The Trans-Omics for Precision Medicine (TOPMed) Program}

We used harmonized phenotype data from eight cohort studies participating in TOPMed (9) Freeze 8 [https://www.nhlbiwgs.org/topmed-whole-genome-sequencing-methods-freeze-8], which included 33,959 genotyped individuals from the Amish Study ( $\square=\square 1,105$ ), JHS (n $\square=\square 2,807$ ), FHS (n $\square=\square 3,658)$, HCHS/SOL $(n=7,693)$, ARIC $(n=7,479)$, CHS $(n=3,482)$, MESA $(n=4,665)$, and CARDIA $(n=3,070)$ with available race/ethnic identification. Descriptions of each of these studies are provided in the Supplementary Materials. This dataset included 8,054 Black participants, 17,143 White participants and 8,762 participants of Hispanic/Latino descent. All participants provided informed consent and the study was approved by IRBs in each of the participating institutions. For TOPMed WGS data acquisition $\quad$ Qnd $\quad$ report $\quad$ see ncbi.nlm.nih.gov/projects/gap/cgi $\square$ bin/GetPdf.cgi?id=phd006969.1. The phenotype harmonization was 
medRxiv preprint doi: https://doi.org/10.1101/2021.09.05.21263126; this version posted September 10, 2021. The copyright holder for this

It is made available under a CC-BY-NC-ND 4.0 International license .

performed by TOPMed Data Coordinating Center (DCC) as described in [29]. The phenotype names and description, exclusion criteria and transformations are described in Supplementary Table 1. Phenotypes' characteristics across race/ethnicities are reported in Supplementary Table 2. All analyses were adjusted for age, sex, study, and reported race/ethnicity as well as 5 first principal components (PCs) to adjust for population structure. The PCs, Kinship matrices, and unrelated individual pools were computed by TOPMed DCC via a robust pipeline [https://github.com/UWGAC/analysis_pipeline] via a combination of KING [47], PC-AiR [53], and PC-Relate[48].

\subsection{The Hispanic Community Health Study/Study of Latinos}

The HCHS/SOL is a community-based cohort study of Hispanic individuals from four field centers across the US [30, 39] with almost 13,000 genotyped participants. A two-stage sampling scheme for participant selection was employed, with sampled community block units followed by households. Correlation matrices to model environmental variance due to households and community block units were generated so that the $i, j$ entry of a given matrix was set to 1 if the $i$ and $j$ individuals live in the same household (or community block unit), and 0 otherwise. This study was approved by the institutional review boards at each field center, where all participants gave written informed consent. Genotyping and quality control for HCHS/SOL have been described in detail elsewhere[53]. In brief, DNA extracted from blood was genotyped on the HCHS Custom 15041502 array (Illumina Omni2.5M + custom content). Genotyping and downstream quality-control procedures yielded 2232944 genetic variants for genotyped HCHS/SOL participants. These were used for genotype imputation with the 1000 Genomes Project phase 3 reference panel [54] as previously described [55]. Variants with at least two copies of the minor allele and present in any of the four 1000 Genomes continental panels were imputed yielding about 50 million imputed variants prior to quality filtering. A subset of 7,693 individuals from HCHS/SOL participated in TOPMed and was used in the first part of this study (with the whole dataset used in the latter parts), with additional phenotypes that were not harmonized across 
medRxiv preprint doi: https://doi.org/10.1101/2021.09.05.21263126; this version posted September 10, 2021. The copyright holder for this preprint (which was not certified by peer review) is the author/funder, who has granted medRxiv a license to display the preprint in perpetuity. It is made available under a CC-BY-NC-ND 4.0 International license .

other TOPMed studies. HCHS/SOL phenotype names and description are described in Supplementary Table 3. The number of participants with non-missing information per phenotype as well as means and standard deviations per phenotype per gender is reported in Supplementary Table 4. We computed genetic correlations between phenotypes from the following domains: anthropometric, blood pressure, lipids, blood cell counts, and inflammation markers. All analyses were adjusted for age, sex, sampling weights and five first principal components to account for population structure.

\subsection{Heritability and Genetic/Environment Correlation estimation}

The relatedness between individuals is modelled via a kinship $(\mathrm{K})$ matrix, and an additional household matrix $(\mathrm{H})$ for modelling environmental effects (available only for the HCHS/SOL cohort). Each phenotype was regressed on age, sex, sampling weights and 5 first principal components (and race/ethnicity and study for TOPMed combined cohorts) and the residuals were rank-normalized. We estimated the correlation coefficients corresponding to the relatedness matrices for all trait pairs by plugging in the normalized residuals to Equation 29. This was implemented via R scripts provided in GitHub repository [https://github.com/tamartsi/HE_Genetic_Correlation]. The genetic and environment variance components as well as the corresponding heritabilities were calculated via the GCTA software [31]. Following sensitivity analysis for the presence of related individuals in the cohorts (Supplementary Figure 2) we removed all individuals related at $3^{\text {rd }}$ degree or more for the calculation of heritabilities, however as we didn't see any significant effects of relatives on the estimated genetic correlations (Supplementary Figure 2B), the relatives were kept in for genetic and environmental correlation coefficients estimation. Bootstrap was used to estimate standard deviations and confidence intervals. Visualizations were performed via the R packages igraph[56], qgraph[57], ggplot2[58] and corrplot[59] followed by Adobe Illustrator. The figures are based on uncorrected p-values. FDRcorrected p-values are provided in the Supplementary Data files. 
medRxiv preprint doi: https://doi.org/10.1101/2021.09.05.21263126; this version posted September 10, 2021. The copyright holder for this preprint (which was not certified by peer review) is the author/funder, who has granted medRxiv a license to display the preprint in perpetuity.

It is made available under a CC-BY-NC-ND 4.0 International license .

\subsection{Domain-Level enrichment analysis}

We have calculated the enrichment of inter-domain correlation via a permutation approach. Specifically for 1000 repeats, we generated random connections between nodes in our correlation graph such that each node will receive a same number of connections as in the real dataset as well as keeping the overall number of connections identical. We then calculated the distribution of number of connections between each pair of domains and used it to obtain a domain enrichment p-value as follows:

$$
\text { p-value (domain } \quad 1, \quad \text { domain } \quad 2) \quad=\frac{1}{1000} \sum_{i=1}^{1000} 1\left(N_{i}^{c}>N^{c}\right)
$$

where $N^{c}$ is the number of connections between domains 1 and 2 , and $N_{i}^{c}, i=1, \ldots, 1000$ is the number of connections between domains 1 and 2 in the $i$ th permutation. We considered two domains to be enriched if their enrichment p-value was $<0.05$.

\section{Author Contributions}

ME, MOG, and TS devised and developed the HE models and framework. ME and TS performed all the analyses, and summarized results in tables and figures. ME and TS conceptualized and drafted the manuscript. TS supervised the work for this manuscript. CI, HC, PSV, HX, AWM, XG, NF, BMP, SSR, JIR, DMLJ, MF, AC, NLHC, RSV, RH, RCS and SR designed data collection and/or TOPMed sample selection in the cohort they represent, formulated best-practices for data analysis for the same cohorts and participated in the manuscript preparation. 
medRxiv preprint doi: https://doi.org/10.1101/2021.09.05.21263126; this version posted September 10, 2021. The copyright holder for this preprint (which was not certified by peer review) is the author/funder, who has granted medRxiv a license to display the preprint in perpetuity.

It is made available under a CC-BY-NC-ND 4.0 International license.

\section{Competing Interests}

B. Psaty serves on the Steering Committee of the Yale Open Data Access Project funded by Johnson \& Johnson. All other co-authors declare no conflict of interest.

\section{Data and Code availability}

TOPMed freeze 8 WGS data are available by application to dbGaP according to the study specific accessions specified in Supplementary Materials. The code is available in GitHub repository [https://github.com/tamartsi/HE_Genetic_Correlation].

\section{References}

1. Pickrell JK, Berisa T, Liu JZ, Ségurel L, Tung JY, Hinds DA (2016) Detection and interpretation of shared genetic influences on 42 human traits. Nat Genet 48:709-717

2. Shi H, Mancuso N, Spendlove S, Pasaniuc B (2017) Local Genetic Correlation Gives Insights into the Shared Genetic Architecture of Complex Traits. Am J Hum Genet 101:737-751

3. van Rheenen W, Peyrot WJ, Schork AJ, Lee SH, Wray NR (2019) Genetic correlations of polygenic disease traits: from theory to practice. Nat Rev Genet 20:567-581

4. Lee SH, Yang J, Goddard ME, Visscher PM, Wray NR (2012) Estimation of pleiotropy between complex diseases using single-nucleotide polymorphism-derived genomic relationships and restricted maximum likelihood. Bioinformatics 28:2540-2542

5. Nagai A, Hirata M, Kamatani Y, et al (2017) Overview of the BioBank Japan Project: Study design and profile. J Epidemiol 27:S2-S8

6. Bycroft C, Freeman C, Petkova D, et al (2018) The UK Biobank resource with deep phenotyping and genomic data. Nature 562:203-209

7. Zheng J, Erzurumluoglu AM, Elsworth BL, et al (2017) LD Hub: A centralized database and 
medRxiv preprint doi: https://doi.org/10.1101/2021.09.05.21263126; this version posted September 10, 2021. The copyright holder for this

preprint (which was not certified by peer review) is the author/funder, who has granted medRxiv a license to display the preprint in perpetuity.

It is made available under a CC-BY-NC-ND 4.0 International license.

web interface to perform LD score regression that maximizes the potential of summary level GWAS data for SNP heritability and genetic correlation analysis. Bioinformatics 33:272-279

8. Sakaue S, Kanai M, Karjalainen J, et al (2020) Trans-biobank analysis with 676,000 individuals elucidates the association of polygenic risk scores of complex traits with human lifespan. Nat Med 26:542-548

9. Kanai M, Akiyama M, Takahashi A, et al (2018) Genetic analysis of quantitative traits in the Japanese population links cell types to complex human diseases. Nat Genet 50:390-400

10. Bulik-Sullivan B, Finucane HK, Anttila V, et al (2015) An atlas of genetic correlations across human diseases and traits. Nat Genet 47:1236-1241

11. Chen MH, Raffield LM, Mousas A, et al (2020) Trans-ethnic and Ancestry-Specific Blood-Cell Genetics in 746,667 Individuals from 5 Global Populations. Cell 182:1198-1213.e14

12. Mogil LS, Andaleon A, Badalamenti A, Dickinson SP, Guo X, Rotter JI, Johnson WC, Im HK, Liu Y, Wheeler HE (2018) Genetic architecture of gene expression traits across diverse populations. PLoS Genet 14:e1007586

13. Burt VL, Whelton P, Roccella EJ, Brown C, Cutler JA, Higgins M, Horan MJ, Labarthe D (1995) Prevalence of hypertension in the US adult population: Results from the third National Health and Nutrition Examination Survey, 1988-1991. Hypertension 25:305-313

14. Rao DC, Sung YJ, Winkler TW, Schwander K, Borecki I, Adrienne Cupples L, James Gauderman W, Rice K, Munroe PB, Psaty BM (2017) Multiancestry Study of Gene-Lifestyle Interactions for Cardiovascular Traits in 610475 Individuals from 124 Cohorts: Design and Rationale. Circ Cardiovasc Genet. https://doi.org/10.1161/CIRCGENETICS.116.001649

15. Walters RK, Polimanti R, Johnson EC, et al (2018) Transancestral GWAS of alcohol dependence reveals common genetic underpinnings with psychiatric disorders. Nat Neurosci $21: 1656-1669$ 
medRxiv preprint doi: https://doi.org/10.1101/2021.09.05.21263126; this version posted September 10, 2021. The copyright holder for this preprint (which was not certified by peer review) is the author/funder, who has granted medRxiv a license to display the preprint in perpetuity.

It is made available under a CC-BY-NC-ND 4.0 International license .

16. Brown BC, Ye CJ, Price AL, Zaitlen N (2016) Transethnic Genetic-Correlation Estimates from Summary Statistics. Am J Hum Genet 99:76-88

17. Galinsky KJ, Reshef YA, Finucane HK, Loh PR, Zaitlen N, Patterson NJ, Brown BC, Price AL (2019) Estimating cross-population genetic correlations of causal effect sizes. Genet Epidemiol 43:180-188

18. Wientjes YCJ, Bijma P, Vandenplas J, Calus MPL (2017) Multi-population genomic relationships for estimating current genetic variances within and genetic correlations between populations. Genetics 207:503-515

19. Schousboe K, Willemsen G, Kyvik KO, et al (2003) Sex Differences in Heritability of BMI: A Comparative Study of Results from Twin Studies in Eight Countries. Twin Res 6:409-421

20. Weiss LA, Pan L, Abney M, Ober C (2006) The sex-specific genetic architecture of quantitative traits in humans. Nat Genet 38:218-222

21. Ober C, Loisel DA, Gilad Y (2008) Sex-specific genetic architecture of human disease. Nat Rev Genet 9:911-922

22. Yang J, Benyamin B, McEvoy BP, et al (2010) Common SNPs explain a large proportion of the heritability for human height. Nat Genet 42:565-569

23. Speed D, Hemani G, Johnson MR, Balding DJ (2012) Improved heritability estimation from genome-wide SNPs. Am J Hum Genet 91:1011-1021

24. Lee SH, Wray NR, Goddard ME, Visscher PM (2011) Estimating missing heritability for disease from genome-wide association studies. Am J Hum Genet 88:294-305

25. Bulik-Sullivan B, Loh PR, Finucane HK, et al (2015) LD score regression distinguishes confounding from polygenicity in genome-wide association studies. Nat Genet 47:291-295

26. Ni G, Moser G, Ripke S, et al (2018) Estimation of Genetic Correlation via Linkage Disequilibrium Score Regression and Genomic Restricted Maximum Likelihood. Am J Hum 
medRxiv preprint doi: https://doi.org/10.1101/2021.09.05.21263126; this version posted September 10, 2021. The copyright holder for this preprint (which was not certified by peer review) is the author/funder, who has granted medRxiv a license to display the preprint in perpetuity.

It is made available under a CC-BY-NC-ND 4.0 International license .

Genet 102:1185-1194

27. Zhang Y, Cheng Y, Jiang W, Ye Y, Lu Q, Zhao H (2020) Comparison of methods for estimating genetic correlation between complex traits using GWAS summary statistics. bioRxiv 2020.10.12.336867

28. Taliun D, Harris DN, Kessler MD, et al (2021) Sequencing of 53,831 diverse genomes from the NHLBI TOPMed Program. Nature 590:290-299

29. Stilp AM, Emery LS, Broome JG, et al (2021) A System for Phenotype Harmonization in the NHLBI Trans-Omics for Precision Medicine (TOPMed) Program. Am J Epidemiol. https://doi.org/10.1093/aje/kwab115

30. Sorlie PD, Avilés-Santa LM, Wassertheil-Smoller S, et al (2010) Design and Implementation of the Hispanic Community Health Study/Study of Latinos. Ann Epidemiol 20:629-641

31. Yang J, Lee SH, Goddard ME, Visscher PM (2011) GCTA: A tool for genome-wide complex trait analysis. Am J Hum Genet 88:76-82

32. Ligthart S, Vaez A, Võsa U, et al (2018) Genome Analyses of >200,000 Individuals Identify 58 Loci for Chronic Inflammation and Highlight Pathways that Link Inflammation and Complex Disorders. Am J Hum Genet 103:691-706

33. Siedlinski M, Jozefczuk E, Xu X, et al (2020) White Blood Cells and Blood Pressure: A Mendelian Randomization Study. Circulation 1307-1317

34. Schillaci G, Pirro M, Pucci G, Ronti T, Vaudo G, Mannarino MR, Porcellati C, Mannarino E (2007) Prognostic Value of Elevated White Blood Cell Count in Hypertension. Am J Hypertens 20:364-369

35. Wainschtein P, Jain DP, Yengo L, et al (2019) Recovery of trait heritability from whole genome sequence data. bioRxiv. https://doi.org/10.1101/588020

36. Reiner AP, Lettre G, Nalls MA, et al (2011) Genome-Wide association study of white blood cell 
medRxiv preprint doi: https://doi.org/10.1101/2021.09.05.21263126; this version posted September 10, 2021. The copyright holder for this preprint (which was not certified by peer review) is the author/funder, who has granted medRxiv a license to display the preprint in perpetuity.

It is made available under a CC-BY-NC-ND 4.0 International license .

count in 16,388 african americans: The continental Origins and Genetic Epidemiology network (COGENT). PLoS Genet 7:e1002108

37. Sodini SM, Kemper KE, Wray NR, Trzaskowski M (2018) Comparison of genotypic and phenotypic correlations: Cheverud's conjecture in humans. Genetics 209:941-948

38. Searle SR (1961) Phenotypic, Genetic and Environmental Correlations. Biometrics 17:474

39. LaVange LM, Kalsbeek WD, Sorlie PD, et al (2010) Sample Design and Cohort Selection in the Hispanic Community Health Study/Study of Latinos. Ann Epidemiol 20:642-649

40. Polderman TJC, Benyamin B, De Leeuw CA, Sullivan PF, Van Bochoven A, Visscher PM, Posthuma D (2015) Meta-analysis of the heritability of human traits based on fifty years of twin studies. Nat Genet 47:702-709

41. Hernandez RD, Uricchio LH, Hartman K, Ye C, Dahl A, Zaitlen N Ultrarare variants drive substantial cis heritability of human gene expression. https://doi.org/10.1038/s41588-019-0487-7

42. Hernandez LM, Blazer DG, Institute of Medicine (US) Committee on Assessing Interactions Among Social B and GF in H (2006) Sex/Gender, Race/Ethnicity, and Health.

43. Talluri R, Shete S (2014) Gaussian graphical models for phenotypes using pedigree data and exploratory analysis using networks with genetic and nongenetic factors based on Genetic Analysis Workshop 18 data. In: BMC Proc. BioMed Central Ltd., p S99

44. Zhao H, Duan ZH (2019) Cancer genetic network inference using gaussian graphical models. Bioinform Biol Insights. https://doi.org/10.1177/1177932219839402

45. Glymour C, Zhang K, Spirtes P (2019) Review of causal discovery methods based on graphical models. Front Genet 10:524

46. Torkamani A, Wineinger NE, Topol EJ (2018) The personal and clinical utility of polygenic risk scores. Nat Rev Genet 19:581-590

47. Manichaikul A, Mychaleckyj JC, Rich SS, Daly K, Sale M, Chen WM (2010) Robust 
medRxiv preprint doi: https://doi.org/10.1101/2021.09.05.21263126; this version posted September 10, 2021. The copyright holder for this preprint (which was not certified by peer review) is the author/funder, who has granted medRxiv a license to display the preprint in perpetuity.

It is made available under a CC-BY-NC-ND 4.0 International license .

relationship inference in genome-wide association studies. Bioinformatics 26:2867-2873

48. Conomos MP, Reiner AP, Weir BS, Thornton TA (2016) Model-free Estimation of Recent Genetic Relatedness. Am J Hum Genet 98:127-148

49. Sofer T (2017) Confidence intervals for heritability via Haseman-Elston regression. Stat Appl Genet Mol Biol 16:259-273

50. Fisher RA (1915) Frequency Distribution of the Values of the Correlation Coefficient in Samples from an Indefinitely Large Population. Biometrika 10:507

51. Bishara AJ, Hittner JB (2017) Confidence intervals for correlations when data are not normal. Behav Res Methods 49:294-309

52. Kunsch HR (1989) The Jackknife and the Bootstrap for General Stationary Observations. Ann Stat 17:1217-1241

53. Conomos MP, Miller MB, Thornton TA (2015) Robust inference of population structure for ancestry prediction and correction of stratification in the presence of relatedness. Genet Epidemiol 39:276-293

54. Altshuler DM, Durbin RM, Abecasis GR, et al (2012) An integrated map of genetic variation from 1,092 human genomes. Nature 491:56-65

55. Conomos MP, Laurie CA, Stilp AM, et al (2016) Genetic Diversity and Association Studies in US Hispanic/Latino Populations: Applications in the Hispanic Community Health Study/Study of Latinos. Am J Hum Genet 98:165-184

56. Csardi G, Nepusz T (2006) The igraph software package for complex network research. InterJournal Complex Sy:1695

57. Epskamp S, Cramer AOJ, Waldorp LJ, Schmittmann VD, Borsboom D (2012) Qgraph: Network visualizations of relationships in psychometric data. J Stat Softw 48:1-18

58. Wickham H (2016) ggplot2: Elegant Graphics for Data Analysis. Springer-Verlag New York 
medRxiv preprint doi: https://doi.org/10.1101/2021.09.05.21263126; this version posted September 10, 2021. The copyright holder for this preprint (which was not certified by peer review) is the author/funder, who has granted medRxiv a license to display the preprint in perpetuity.

It is made available under a CC-BY-NC-ND 4.0 International license .

59. Wei T, Simko V (2021) R package "corrplot": Visualization of a Correlation Matrix. 